\title{
Driver perceptions of advanced driver assistance systems: A
}

\section{case study [version 1; peer review: awaiting peer review]}

\author{
Siti Fatimah Abdul Razak (D1, Sumendra Yogarayan (D1), Afizan Azman², \\ Mohd Fikri Azli Abdullah¹, Anang Hudaya Muhamad Amin (ii) 3 , Mazrah Salleh4
}

\author{
${ }^{1}$ Multimedia University, Ayer Keroh, Melaka, 75450, Malaysia \\ ${ }^{2}$ Kolej Universiti Islam Melaka, Kuala Sg. Baru, Melaka, Malaysia \\ ${ }^{3}$ Higher Colleges of Technology, Dubai, United Arab Emirates \\ ${ }^{4}$ Rolls-Royce, Derby, England, UK
}

V1 First published: 08 Nov 2021, 10:1122
https://doi.org/10.12688/f1000research.73400.1

Latest published: 08 Nov 2021, 10:1122

https://doi.org/10.12688/f1000research.73400.1

\begin{abstract}
Background: Automobile manufacturers need to have an insight and understand how consumers, specifically drivers, respond to the advanced driver assistance systems (ADAS) technology in their manufactured vehicles. This study reveals drivers' perceptions of Malaysia's advanced driver assistance systems, which is currently lacking in the literature. So far, other studies have focused on countries that are unlike Malaysia's multi-culture environment. Methods: A survey was designed and distributed using convenience sampling to obtain responses from licensed drivers. Questions included demographic and driving questions, the perceptions of benefits and obstacles relevant to ADAS use, vehicle decision-making, and technology use. Data were collected from 818 respondents who were licensed drivers in Malaysia. Results were then analysed using statistical approaches.

Results: The findings indicated that $76.8 \%$ of drivers have a positive attitude towards ADAS technology, particularly safety applications when they are available. Regardless of the accuracy of these systems, acceptance of the technology may shift upon viewing or hearing messages of possible problems with ADAS.

Conclusions: It can be concluded that the safety advantages of ADAS technology are less valued by drivers who do not have experience of road traffic accidents. Furthermore, acceptance of the technology could be undermined by assuming that the safety applications could be compromised.
\end{abstract}

Keywords

safety applications, road safety, collision avoidance, ADAS, driver

perceptions, vehicle technology

\section{Open Peer Review}

Approval Status AWAITING PEER REVIEW

Any reports and responses or comments on the article can be found at the end of the article. 


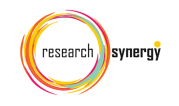

sis.

This article is included in the Research Synergy

Foundation gateway.

Corresponding author: Siti Fatimah Abdul Razak (fatimah.razak@mmu.edu.my)

Author roles: Abdul Razak SF: Conceptualization, Data Curation, Formal Analysis, Methodology, Writing - Original Draft Preparation, Writing - Review \& Editing; Yogarayan S: Conceptualization, Data Curation, Writing - Original Draft Preparation, Writing - Review \& Editing; Azman A: Investigation, Project Administration, Resources, Validation; Abdullah MFA: Investigation, Methodology, Validation; Muhamad Amin AH: Methodology, Validation, Writing - Review \& Editing; Salleh M: Validation, Visualization

Competing interests: No competing interests were disclosed.

Grant information: This work is partially supported by the Ministry of Higher Education Fundamental Research Grant Scheme, MMUE 190034/1/2019.

Copyright: ( 2021 Abdul Razak SF et al. This is an open access article distributed under the terms of the Creative Commons Attribution License, which permits unrestricted use, distribution, and reproduction in any medium, provided the original work is properly cited.

How to cite this article: Abdul Razak SF, Yogarayan S, Azman A et al. Driver perceptions of advanced driver assistance systems: $\mathbf{A}$ case study [version 1; peer review: awaiting peer review] F1000Research 2021, 10:1122

https://doi.org/10.12688/f1000research.73400.1

First published: 08 Nov 2021, 10:1122 https://doi.org/10.12688/f1000research.73400.1 


\section{Introduction}

Road safety is a collective responsibility involving public and private corporations and civil society ${ }^{1}$. In recent years, automobile manufacturers have provided in-vehicle or pre-installed Advanced Driver Assistance Systems (ADAS) and parking control $^{2}$ to support three types of driving tasks: navigation, manoeuvring, and vehicle stabilisation. These systems were typically developed for the global market without considering cultural differentiation, influencing drivers' attitudes and acceptance ${ }^{3}$. The perception of new technology will naturally vary depending on locality, and it is primarily dictated by individual-level factors such as age, experience and knowledge of the technology ${ }^{4}$. Hence, it is crucial to evaluate the response of individuals from specific cultural backgrounds ${ }^{5}$. To the best of our knowledge, a study to better understand Malaysian licensed driver's general acceptance of ADAS technology has yet to be carried out. The technology currently being offered is vehicleresident systems, and we believe the knowledge gap regarding these systems is worth filling.

Hence, this paper reports a survey to assess whether drivers are willing to adopt and use ADAS technology. Our specific objectives were to explore the perceived importance and benefits associated with the technology and the influence of driving behaviour on the willingness of drivers to adopt the technology.

\section{Methods}

Participants

In this study, we invited 1000 individuals to participate as respondents using convenience sampling, a non-probability sampling often associated with this type of study. The authors of this survey, who met the eligibility criteria, were excluded as potential participants.

\section{Recruitment}

The survey was accessible to the respondent who received an email invitation. We contacted individuals with email addresses who might contribute to our survey. Participants and colleagues were encouraged to recommend additional potential survey participants. The survey was not promoted online.

\section{Survey administration}

The survey was administered by inviting respondents to fill out an online survey investigating their opinions about transportation and ADAS technology. A link to the Google Form survey was included in the invitation emails. Invitations were sent out for a duration of one month, starting 20th March 2020. Respondents were encouraged to provide feedback in a single session to avoid distractions. After three days, respondents who had not responded were sent a reminder email. A second reminder was sent if no response was received within seven days. If no response was received, the respondent was deemed disinterested. Responses could be modified before submission but respondents could not re-enter the form using the same email address after it was submitted. There were no incentives offered to respondents for completing the survey.

\section{Survey instrument}

The survey instrument was adapted from 6 to tap factors affecting acceptance of ADAS technology. The survey instrument was designed to assess the acceptance rate of Malaysian licensed drivers towards ADAS technology.

The participants had to answer 20 closed-ended or rating-scale questions. The questions were divided into three sections: Section A, Section B, and Section C. First, respondents answered demographic and driving-related questions. Next, respondents were asked about their views of the advantages and challenges of utilising ADAS technology. Finally, respondents were queried on vehicle decision-making and technology use. To prevent distractions and higher turnover, they were also encouraged to keep survey sessions to 20 minutes or less. Respondents who rated three or higher for rating-based questions were classified as "ADAS accepters."

\section{Data analysis}

This case study was not intended to make statistical inferences concerning the wider population. Respondents who were Malaysian licensed drivers had higher merit than others. The underlying data from this study is accessible from Abdul Razak et al., 7 . We used descriptive statistics to analyse the ratings given by the respondents on each item. Microsoft Excel (Microsoft Excel, RRID: SCR_016137) was used for this purpose.

\section{Ethical approval}

The Technology Transfer Office of the Multimedia University ethically approved this research. (Approval Number: EA0562021). In the invitation email, the survey participants were informed that (i) any information given was voluntary; (ii) the researchers were not accountable or responsible for any loss arising from the use of the information provided; (iii) the survey was expected to take 20 minutes; (iv) any identifying information acquired in connection with this survey would remain confidential; and (v) the findings may be submitted for publication, and the anonymised dataset would be made publicly available in a data repository. The original dataset was password-protected in a Google Drive folder.

\section{Reporting guidelines}

Reporting Qualitative Research $(\mathrm{SPQR})^{8}$ were adhered to to report the survey outcomes.

\section{Results}

Out of the invited 1000 individuals, $81.80 \%$ completed the survey. Out of these respondents, $92.42 \%$ were licensed drivers. The majority of respondents were 18 to 25 years old, including newly licenced drivers, students, and employees. For daily tasks or routines, these responders usually drove their own or a family member's vehicle. The least represented group was those in the 55 years old and above range. About $45 \%$ of the respondents were adults in the 26 years and above age range. The distribution of male and female respondents was almost similar, marginally favouring males (Table 1). Over half of the respondents resided in urban and suburban areas. 
Table 1. Demographics of respondents.

\begin{tabular}{|c|c|c|c|c|}
\hline \multirow{2}{*}{ Age group (years) } & \multicolumn{2}{|c|}{ Gender } & \multicolumn{2}{c|}{ Own Malaysia driving license } \\
\hline & Male & Female & Male & Female \\
\hline $18-25$ & 291 & 157 & 269 & 134 \\
\hline $26-34$ & 78 & 72 & 75 & 65 \\
\hline $35-54$ & 62 & 94 & 60 & 90 \\
\hline 55 and above & 36 & 28 & 35 & 28 \\
\hline Total & $467(57.09 \%)$ & $351(42.91 \%)$ & $439(58.10 \%)$ & $317(41.93 \%)$ \\
\hline
\end{tabular}

\section{Driving behaviour}

We found that $10 \%$ of respondents had not driven since receiving their driving licence. Hence their driving behaviour was not analysed. Of those who drove, 50\% resided in urban areas, $54.44 \%$ of respondents reported travelling less than $400 \mathrm{~km}$ daily for different reasons, while $44 \%$ claimed involvement in accidents previously (Table 2). However, of those involved in accidents, $58.49 \%$ were only involved in minor accidents.

The impact of the accident was further investigated. We asked if the accident caused major or minor damage to the vehicle involved and if the driver or passenger required medical attention. It could also have been a combination of any of these three factors. The respondents stated that $23.62 \%$ experienced major damage, which cost them more than RM1000 to repair their vehicles, whereas $53.27 \%$ were involved in minor accidents. Around $9 \%$ of the respondents were involved in more than one accident, which resulted in experiencing more than one of the factors mentioned earlier.

\section{Acceptance}

Respondents were categorised as non-accepters, fence-sitters, and accepters based on their responses to a willingness to have in-vehicle systems providing driving assistance. Almost 82\% of respondents were receptive to the technology (accepters), while $16.99 \%$ of respondents were non-receptive. Only $1.03 \%$ were fence-sitters. The accepters were younger drivers who were more receptive to new technology (see Table 3). Nevertheless, older drivers who had been involved in accidents previously were also receptive to the technology. Non-accepters required more information on the benefits of the technology and had a wait-and-see attitude before accepting the technology.

\section{Importance}

Respondents were further requested to rate safety applications, which they perceived as crucial for their driving experience in terms of convenience, economy, and safety. This included self-driving systems, lane changing, automated braking, and collision avoidance, signal timing, or blind spot occupancy, as well as real-time information related to routes, congestion, and incidents as part of their driving experience. Figure 1 illustrates the importance, rated as extremely important, very important, moderately important, slightly important, and not important.

\section{Adoption preference}

Of the respondents, $82.7 \%$ favoured having Advanced Driver Assistance Systems, which can assist driving. Nevertheless, $48.9 \%$ of the respondents would like to wait until the technology has been thoroughly tested and checked before using Advanced Driver Assistance Systems. They would also be more confident about embracing the technology if they could see their associates, i.e., people they know using it. A total of $18.9 \%$ also tended to wait until the latest product excitement had subsided before the technology was purchased and used. However, almost a quarter $(24.3 \%)$ of the respondents preferred to be the first to buy and try the new technology. About $7.8 \%$ of the respondents were not sure of their purchasing decision behaviour. This may be attributed to influence from family members, where $68.7 \%$ of the respondents indicated that family members influence their vehicle purchasing decision. However, $76.8 \%$ of the respondents would be willing to use the technology if it was pre-installed by the vehicle manufacturers. The remaining respondents would either ignore, disable, or remove the technology from the vehicle. About half of the respondents were willing to add Advanced Driver Assistance Systems to their vehicles if the cost was less than RM400.

\section{Concerns and perceived benefits}

The respondents' concerns and perceived benefits relating to the implementation of ADAS technology in personal vehicles can be categorised into three groups: driver behaviour, security or safety, technology implementation.

Driver behaviour relates to how the respondents react as drivers and how other drivers react. Of the respondents, 55\% feared that over-reliance on technology or other diversions might make drivers less attentive. Drivers frequently depend on technology too much and don't practice safe driving (e.g., maintain a safe distance from other vehicles). A total of $30 \%$ of respondents were worried about vehicle data security breaches allowing illegal activities and affecting their driving habits. Moreover, the electromagnetic activity of communication devices, such as those in the system, may endanger drivers' health. Nevertheless, $15 \%$ of respondents were eager to adopt ADAS technology for a safer driving experience. 
Table 2. Involvement in accidents.

\begin{tabular}{|c|c|c|c|}
\hline \multirow{2}{*}{ Accidents involvement (years ago) } & \multicolumn{4}{|c|}{ Residential area } \\
\hline & Urban & Suburban & Rural \\
\hline 5 & 95 & 63 & 36 \\
\hline $5-10$ & 27 & 12 & 11 \\
\hline $11-15$ & 7 & 6 & 5 \\
\hline $16-20$ & 10 & 5 & 3 \\
\hline$>20$ & 9 & 4 & 1 \\
\hline 0 (never) & 184 & 94 & 91 \\
\hline
\end{tabular}

\section{Table 3. Acceptance of technology.}

\begin{tabular}{|c|c|c|c|c|c|c|}
\hline \multirow{2}{*}{ Age (years) } & \multicolumn{2}{|c|}{ Non-accepters } & \multicolumn{2}{c|}{ Accepters } & \multicolumn{2}{c|}{ Fence-sitters } \\
\hline & Acc.= Yes & Acc. $=$ No & Acc.= Yes & Acc.= No & Acc.= Yes & Acc.= No \\
\hline $18-25$ & 22 & 36 & 99 & 191 & 1 & 2 \\
\hline $26-34$ & 6 & 15 & 49 & 55 & 1 & 1 \\
\hline $35-54$ & 11 & 17 & 67 & 44 & 0 & 2 \\
\hline 55 and above & 7 & 1 & 38 & 12 & 0 & 0 \\
\hline & $115(16.99 \%)$ & $555(81.98 \%)$ & $7(1.03 \%)$ \\
\hline
\end{tabular}

$A c c .=$ Yes: respondents who have experienced accidents

Acc. $=$ No: respondents who have never been in accidents

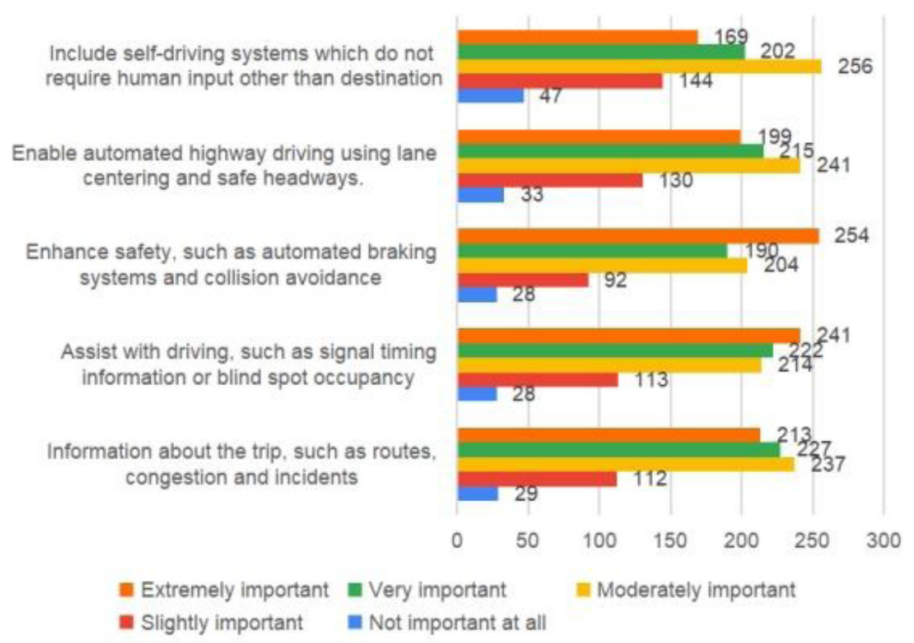

Figure 1. Perceived importance of Advanced Driver Assistance Systems.

\section{Discussion}

Generally, consumer perceptions vary depending on age, experience, and technology knowledge ${ }^{4}$. Our results complement the findings of earlier studies where drivers acceptance of ADAS technology was the main focus but different populations and cultures were examined such as the Czech Republic ${ }^{9}$, 
Finland ${ }^{10}$ and Indonesia ${ }^{11}$. This research found that $76.8 \%$ of drivers favoured ADAS technology, especially safety applications. This is higher than a similar study among Czech drivers, which reported that ADAS technology was desired by at least $50 \%$ of the respondents ${ }^{9}$. However, the majority were only ready to utilise the technology when it was available and free. Individual variables like experience may also affect drivers' adoption of the technology. This is supported by our analysis that found that ADAS technology's safety advantages are less valued by drivers who do not experience road traffic accidents. However, $44 \%$ of drivers who have never been in an accident could be the future technology acceptors.

The study that investigated Finnish drivers focused on drivers who drive regularly and have purchased new cars with adaptive cruise control (ACC) and lane keeping assist (LKA). Another study among Jakarta drivers focused on drivers acceptance of the forward collision warning and lane departure warning ${ }^{11}$. However, this research looked at drivers' acceptance of ADAS technology in general, dividing them into three categories: acceptors, fence-sitters, and non-acceptors. Most drivers are unaware that the technology is available in recent vehicle models. Thus, their contribution is unaffected by their present technology usage.

Moreover, to enhance the perceived self-risk and the importance of safety applications, communications with this group of drivers should discuss the prevalence of accidents. This group of drivers may respond to messages about the costs of even minor accidents. The advantages of using safety technology should be highlighted, including saving lives and avoiding serious injury. Regardless of the accuracy of these results, acceptance of the technology may shift upon viewing or hearing messages of possible problems with ADAS technology. Hence, the realistic goal for automotive manufacturers is to reach the majority of Malaysian drivers and thereby stimulate the development of ADAS technology to reduce the likelihood of road traffic accidents.

Communication strategies are often designed to overcome potential challenges. For instance, safety and security issues were reported by $33 \%$ percent of the respondents as very important. The respondents may think that the safety applications could be compromised, and related agencies should educate drivers about misconceptions with fact sheets, by using videos and exchanging expert opinions. Evidence and anecdotes from victims of traffic accidents or early technology users are also likely to be helpful.

Most participants in the survey (76.8\%) mentioned they would use the safety application system or ADAS technology in a purchased or leased vehicle. Presumably, if the systems were introduced in fleets such as taxis, e-hailing services, and rental cars, the systems would likely be more common, giving consumers a chance to embrace technology.

To summarise, the research investigated Malaysian drivers' expectations concerning ADAS technology. Those who have never been in a road accident may be less interested in the ADAS technology's safety benefits. It is feasible to anticipate the market's future by analysing driver acceptance of ADAS technology, particularly in countries with comparable cultural variety to Malaysia. Thus, ADAS technology should be assessed often to determine which systems have the most potential to improve driver safety.

\section{Conclusions}

This paper aimed to fill a gap in the literature by examining drivers' views of Advanced Driver Assistance Systems (ADAS) in Malaysia. Prior research has concentrated on countries that are not comparable to Malaysia's multi-cultural context. Driver acceptance of ADAS technology is gradual because there are limited interactions or connectivity between systems functionalities and drivers via human-machine interface in the vehicle context. Thus, if ADAS technology aims to improve driver's safety, much research remains to be done.

\section{Data availability}

Underlying data

Zenodo: Underlying data for 'Driver perceptions on Advanced Driver Assistance Systems: A case study', https://doi.org/10.5281/ zenodo. 5547222

This project contains the following underlying data:

i. V2V safety technology survey (responses).xlsx

ii. V2V safety technology survey (processed).xlsx

iii. V2V safety technology survey_GoogleForm.pdf

Data are available under the terms of the Creative Commons Attribution 4.0 International license (CC-BY 4.0).

\section{Consent}

Written informed consent for publication of the participants' details was obtained from the participants.

\section{Author contributions}

S.F.A.R., S.Y., A.A., M.F.A.A., A.H.M.A, and M.S. comprehended the idea and contributed to the research article. All authors contributed to the writing, editing, and consent of the final manuscript.

\section{Acknowledgements}

The authors would like to thank everyone who has directly or indirectly contributed towards this research. We thank the anonymous reviewers for their constructive comments. 
1. Eusofe $Z$, Evdorides $H$ : Assessment of road safety management at institutional level in Malaysia: A case study. IATSS research. 2017; 41(4): 172-181.

Publisher Full Text

2. Haboucha $\mathrm{CJ}$, Ishaq R, Shiftan $\mathrm{Y}$ : User preferences regarding autonomous vehicles. Transport Res C-Emer. 2017; 78: 37-49.

Publisher Full Text

3. Large DR, Burnett G, Mohd-Hasni Y: Capturing cultural differences between UK and Malaysian drivers to inform the design of in-vehicle navigation systems. Int J Auto Eng. 2017; 8(3): 112-119. Publisher Full Text

4. Moody J, Bailey N, Zhao J: Public perceptions of autonomous vehicle safety: An international comparison. Safety Sci. 2020; 121: 634-650. Publisher Full Text

5. Kassim KA, Nasruddin MA, Jawi ZM: Assessing the Public Opinion on Autonomous Vehicles in Malaysia. J Soc Auto Malay. 2019; 3(2). Reference Source

6. Levine E, Brennan E, Garfield K, et al.: Vehicle-to-Vehicle crash avoidance technology: public acceptance final report. (No. FHWA-JPO-17-491). 2015. Reference Source

7. Abdul Razak SF, Yogarayan S, Azman A, et al.: Driver Perceptions on Advanced Drive Assistance Systems. [Data set]. Zenodo. 2021.

http://www.doi.org/10.5281/zenodo.5547222

8. O'Brien BC, Harris IB, Beckman TJ, et al.: Standards for reporting qualitative research. Acad Med. 2014; 89(9): 1245-51.

Publisher Full Text

9. Viktorova L, Sucha M: Drivers' acceptance of advanced driver assistance systems- what to consider? International Journal of Traffic and Transport Engineering. 2018; 8(3): 320-333. Publisher Full Text

10. Pentinnen M, Louma J: Acceptance and use of ADAS. Proceedings of TRA2020 the 8th Transport Research Arena. 2020; 1-10. Reference Source

11. Zaki A, Suzianti A, Ardi R: Assessing Driver Acceptance of Jakarta Community Towards FCW and LDW. 2019 4th International Conference on Intelligent Transportation Engineering (ICITE). 2019; 109-114. Publisher Full Text 
The benefits of publishing with F1000Research:

- Your article is published within days, with no editorial bias

- You can publish traditional articles, null/negative results, case reports, data notes and more

- The peer review process is transparent and collaborative

- Your article is indexed in PubMed after passing peer review

- Dedicated customer support at every stage

For pre-submission enquiries, contact research@f1000.com 